\title{
Preparation and Photoluminescence Spectra of Organometallic Complexes Containing Nanoparticles as Random Gain Media
}

\author{
Atheer Abdulraheem Mahmood ${ }^{{ }^{*}}$, Oday Atta Hammadi ${ }^{2}$, and Kais Rzaik Ibraheem ${ }^{3}$ \\ ${ }^{1}$ Department of Chemistry, College of Education, Al-Iraqia University, Baghdad, Iraq \\ ${ }^{2}$ Department of Physics, College of Education, Al-Iraqia University, Baghdad, Iraq \\ ${ }^{3}$ Department of Chemistry, College of Science, University of Anbar, Ramadi, Iraq
}

*Corresponding author:

tel: $+964-7813479524$

email: atheerinchemistry@yahoo.com

Received: September 1, 2021

Accepted: November 20, 2021

DOI: $10.22146 / \mathrm{ijc} .68822$

\begin{abstract}
This work prepared organometallic complexes from the 8-hydroxyquinoline ligand linked to metal ions such as $\mathrm{Ba}^{2+}, \mathrm{Ca}^{2+}$, and $\mathrm{Zn}^{2+}$. The effects of metal ion type and adding nanoparticles to the complex solution on the absorption and photoluminescence characteristics of the prepared complexes were introduced. These nanoparticles were added to the prepared complex solutions to act as scattering centers to form random gain media with emission in the visible region of the electromagnetic spectrum. The random gain media made from the $\mathrm{Znq}_{2}$ complex with nanoparticles showed the best characteristics with good chemical and spectroscopic stabilities, high reliability, and reproducibility in addition to the low production cost and reasonably simple requirements.
\end{abstract}

Keywords: photoluminescence; organometallic complex; nanoparticles; random gain medium

\section{- INTRODUCTION}

As of late, different interchanges, natural, biomedical, and space applications have been created by using irregular addition media as laser sources known as "random lasers" [1]. Additionally, the continuous and extraordinary improvements in nanotechnology have prompted uncommon advancements in the plan and manufacture of irregular increase media [2-3]. 8Hydroxyquinoline zinc has many advantages, such as simple synthesis conditions [4]. In general, the random gain medium can be fabricated by adding nanoparticles to a laser dye solution to play the role of scatters within the dye solution to amplify the generated laser signal [5]. However, the spectroscopic characteristics of both dye solution and nanoparticles must be precisely determined as a requirement for successful gain amplification.

As the power of the random lasing activity is amazingly high, then, at that point, the population inversion is reached so rapidly, bringing about a short peak in the fluorescence spectrum. The population inversion is effectively achieved and consumed with continuous excitation of the added particles, prompting a progression of peaks in the emission range. The emission range of a random laser is high in intensity and narrow in width.

The host or gain media used to fabricate random laser systems are laser dyes like xanthene and coumarin. These dyes are characterized by high gain, high fluorescence efficiency, and extremely broad energy bands allowing both absorption and emission over a wide range of wavelengths. Also, the emission of laser dye can be controlled by several parameters, mainly dyesolution concentration, and solvent type. Therefore, the search for new organic complexes with featured spectroscopic and chemical characteristics continuously replaces common laser dyes and explores new dye laser physics and technology [6]. Znq 2 has many advantages, such as high luminous efficiency [6]. Many scholars have done in-depth research focused on extending application potential of $\mathrm{MQ}_{2}$ [7]. At present, liquidphase method is the main method to synthesize $\mathrm{MQ}_{2}$ [8].

The 8-hydroxyquinoline (H.Q.) ligand is impressively used in coordination science for the extraction and goal of component particles [9-10]. 
Chelato-aromaticity can happen in metallo-cyclic rings shaped during metal particle complexation or in rings of the ligand that do not connect straightforwardly with metal particles [11]. The 8-hydroxyquinoline can arrange with different particles as bidentate through nitrogen iota of quinolone ring and oxygen molecule after deprotonation of hydroxyl group [12]. The 8-hydroxyquinoline (oxine) acts as a bidentate $\left(\mathrm{N}, \mathrm{O}^{-}\right)$univalent ligand to frame chelates with a few metal particles [13]. The zinc(II) buildings of amide and urea subbed of 8-hydroxyquinoline have shown superb photoluminescence and fluorescence qualities compared to their friend metalorganic edifices [14].

This work synthesized new organometallic complexes by connecting some metal ions to the 8hydroxyquinoline ligand. The photoluminescence of the incorporated complexes was estimated before and after adding metal oxide nanoparticles to introduce their effects and form a random gain media to produce a random laser.

\section{- EXPERIMENTAL SECTION}

\section{Materials}

Three different solvents, dimethyl sulfoxide (DMSO), N,N-dimethylformamide (DMF), and ethanol, were used in this work, while the 8-Hydroxyquinoline (8HQ) was used as a ligand and barium(II) chloride dihydrate, calcium(II) chloride and zinc (II) chloride were used as metal salts to synthesize the required complexes.

\section{Instrumentation}

The precursors, ligands, and synthesized complexes were characterized as follows. The Fourier-transform infrared (FTIR) spectra were recorded using the Nicolet Impact 410 spectrophotometer using $\mathrm{KBr}$ disk in the range $400-4000 \mathrm{~cm}^{-1}$. The absorption spectra were recorded using a UV-Visible SPEKOL 2000 double-beam spectrophotometer supplied by P.G. Instruments (U.K.), which has a slit width in a spectral range of 190-1100 nm. Both FTIR and UV-visible measurements were performed on the samples in ethanol. The field-effect scanning electron microscopy (FE-SEM) was used to study the effect of nanoparticle size and distribution on the characteristics of the prepared samples.

\section{Procedure}

\section{Synthesis of bis(8-hydroxyquinolato) zinc $\left(\mathrm{Znq}_{2}\right)$ complex}

A sample of $1.83 \mathrm{~g}(10 \mathrm{mmol}) \mathrm{Zn}\left(\mathrm{CH}_{3} \mathrm{COO}\right)_{2}$ was dissolved in $10 \mathrm{~mL}$ of distilled water. Then this solution was added as drops to another solution of $2.9 \mathrm{~g}(2 \mathrm{mmol})$ 8-hydroxyquinoline ligand was dissolved in a mixture of $0.11 \mathrm{~g}$ potassium hydroxide $(\mathrm{KOH})$ in $6 \mathrm{~mL}$ of distilled water. The first solution prepared in the first step was added to the second solution, and then the reaction mixture was stirred as the complex was prepared by filtration, washed by absolute ethanol, dried at room temperature for $24 \mathrm{~h}$. The result is a white-green solid with a yield of $3.26 \mathrm{~g}(92.35 \%)$.

\section{Synthesis of bis(8-hydroxyquinolato) barium (Baq 2$)$ complex}

A $2.44 \mathrm{~g}(10 \mathrm{mmol})$ of $\mathrm{BaCl}_{2} \cdot 2 \mathrm{H}_{2} \mathrm{O}$ was dissolved in $10 \mathrm{~mL}$ distilled water, then the prepared solution was added to a solution of $2.9 \mathrm{~g} \mathrm{(} 2 \mathrm{mmol}$ ) 8-HQ dissolved in a solution of $0.11 \mathrm{~g} \mathrm{KOH}$ in $6 \mathrm{~mL}$ of distilled water. The first solution was added to the second solution, and the mixture was stirred until the complex was prepared by filtration, washed by absolute ethanol, dried at room temperature for $24 \mathrm{~h}$ to get a white green solid with a yield of $4.08 \mathrm{~g}(81.6 \%)$.

\section{Synthesis of bis(8-hydroxyquinolato) calcium ( $\left.\mathrm{Caq}_{2}\right)$ complex}

A solution was prepared by dissolving $1.11 \mathrm{~g}$ (10 mmol) $\mathrm{CaCl}_{2}$ in $10 \mathrm{~mL}$ distilled water, then added to

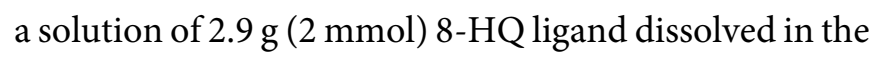
same mixture $(0.11 \mathrm{~g} \mathrm{KOH}$ in $6 \mathrm{~mL}$ of distilled water). The first solution was added to the second one, and the produced mixture was stirred until the complex was prepared by filtration, washed by absolute ethanol, dried at room temperature for $24 \mathrm{~h}$. The final sample was a white-yellow solid with a yield of $1.82 \mathrm{~g}(98.7 \%)$.

\section{Adding nanoparticles}

In order to form the random gain media, highlypure titanium dioxide nanoparticles with an average particle size of $25 \mathrm{~nm}$ were added to the complex solution, and the absorption and photoluminescence spectra were recorded and compared before and after 
adding these nanoparticles. The minimum amount of added nanoparticles was $0.5 \mathrm{mg}$ for $5 \mathrm{~mL}$ of a complex solution of $10^{-5} \mathrm{M}$ concentration. Many experiments were carried out to determine these preparation conditions.

\section{- RESULTS AND DISCUSSION}

To affirm the arrangement of organometallic linkage, the FTIR spectra of the free 8-hydroxyquinoline ligand and all edifices arranged in this work were recorded in the wavenumber scope of $400-4000 \mathrm{~cm}^{-1}$ as displayed in Fig. 1. The range of the 8-Hq ligand shows two groups at $3161 \mathrm{~cm}^{-1}$ because of the $\mathrm{O}-\mathrm{H}$ extending group and at $3049 \mathrm{~cm}^{-1}$ to C-Hst, group aromatic vibration. The main band was not found in the FTIR spectra of the three complexes, which affirms the coordination through the oxygen atom of the hydroxyl group. The subsequent band was seen inside the reach $3040-3055 \mathrm{~cm}^{-1}$.

One more band at $1576 \mathrm{~cm}^{-1}$ attributed to the $\mathrm{C}=\mathrm{N}$ group was marginally moved to various positions, which affirms the coordination through the nitrogen iota of the $\mathrm{C}=\mathrm{N}$ group [15]. The groups were seen at 1500 and $1466 \mathrm{~cm}^{-1}$ are ascribed to the vibrations of the benzene ring. These bands were seen in all spectra of the prepared complexes with a slight shift, as shown in Table 1 . The bands are seen within the range $1366-1386 \mathrm{~cm}^{-1}$ are ascribed to the vibration of $\mathrm{C}=\mathrm{N}$. The bands seen in the range $1274-1281 \mathrm{~cm}^{-1}$ are attributed to $\mathrm{O}-\mathrm{H}$ 's bend vibrations [16-17]. The bands observed at 1217 and
$1203 \mathrm{~cm}^{-1}$ are ascribed to the stretch vibration modes of $\mathrm{C}-\mathrm{O}$.

Nonetheless, these groups were seen in the FTIR spectra of the $\mathrm{Baq}_{2}$ complex and disappeared in the $\mathrm{Caq}_{2}$ and $\mathrm{Znq}_{2}$. Therefore, the new groups have seen inside $570-591 \mathrm{~cm}^{-1}$ and $475-504 \mathrm{~cm}^{-1}$ are ascribed to the arrangement of metal-nitrogen $(\mathrm{M}-\mathrm{N})$ metal-oxygen (M-O) bonds, separately, because of the coordination with metal particles in the buildings $\left(\mathrm{Baq}_{2}, \mathrm{Caq}_{2}\right.$, and $\mathrm{Znq}_{2}$ ) [18-20].

Fig. 2 shows the absorption spectra of the three complexes prepared in this work before and after adding

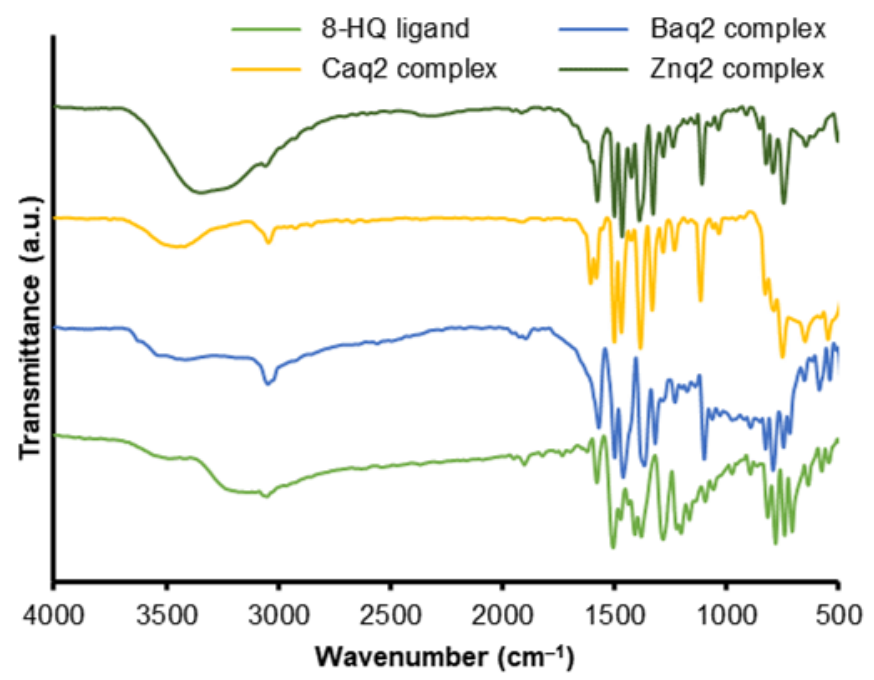

Fig 1. FTIR spectra of free 8-Hq ligand, $\mathrm{Baq}_{2}, \mathrm{Caq}_{2}$, and $\mathrm{Znq}_{2}$ complexes prepared in this work

Table 1. Vibration modes of the free ligand and complexes prepared in this work

\begin{tabular}{lcccc}
\hline Vibration mode & $8-\mathrm{Hq}\left(\mathrm{cm}^{-1}\right)$ & $\mathrm{Baq}_{2}\left(\mathrm{~cm}^{-1}\right)$ & $\mathrm{Caq}_{2}\left(\mathrm{~cm}^{-1}\right)$ & $\mathrm{Znq}_{2}\left(\mathrm{~cm}^{-1}\right)$ \\
\hline M-O vibration & 475 & 478 & 492 & 504 \\
M-N vibration & 577 & 584 & 591 & 570 \\
In-plane ring deformation & 642 & 645 & 648 & 644 \\
In-plane deformation & 743 & 740 & 748 & 742 \\
C-O stretching & 1203 & 1198 & 1171 & 1175 \\
O-H bending & 1281 & 1281 & 1274 & 1280 \\
C=N vibration & 1375 & 1366 & 1375 & 1386 \\
Phenyl group & 1466 & 1460 & 1459 & 1466 \\
Pyridyl group & 1500 & 1497 & 1497 & 1500 \\
C=N coordination & 1576 & 1567 & 1569 & 1577 \\
C-H $H_{\text {st. }}$ aromatic vibration & 3049 & 3044 & 3040 & 3054 \\
OH stretching & 3161 & - & - & - \\
\hline
\end{tabular}



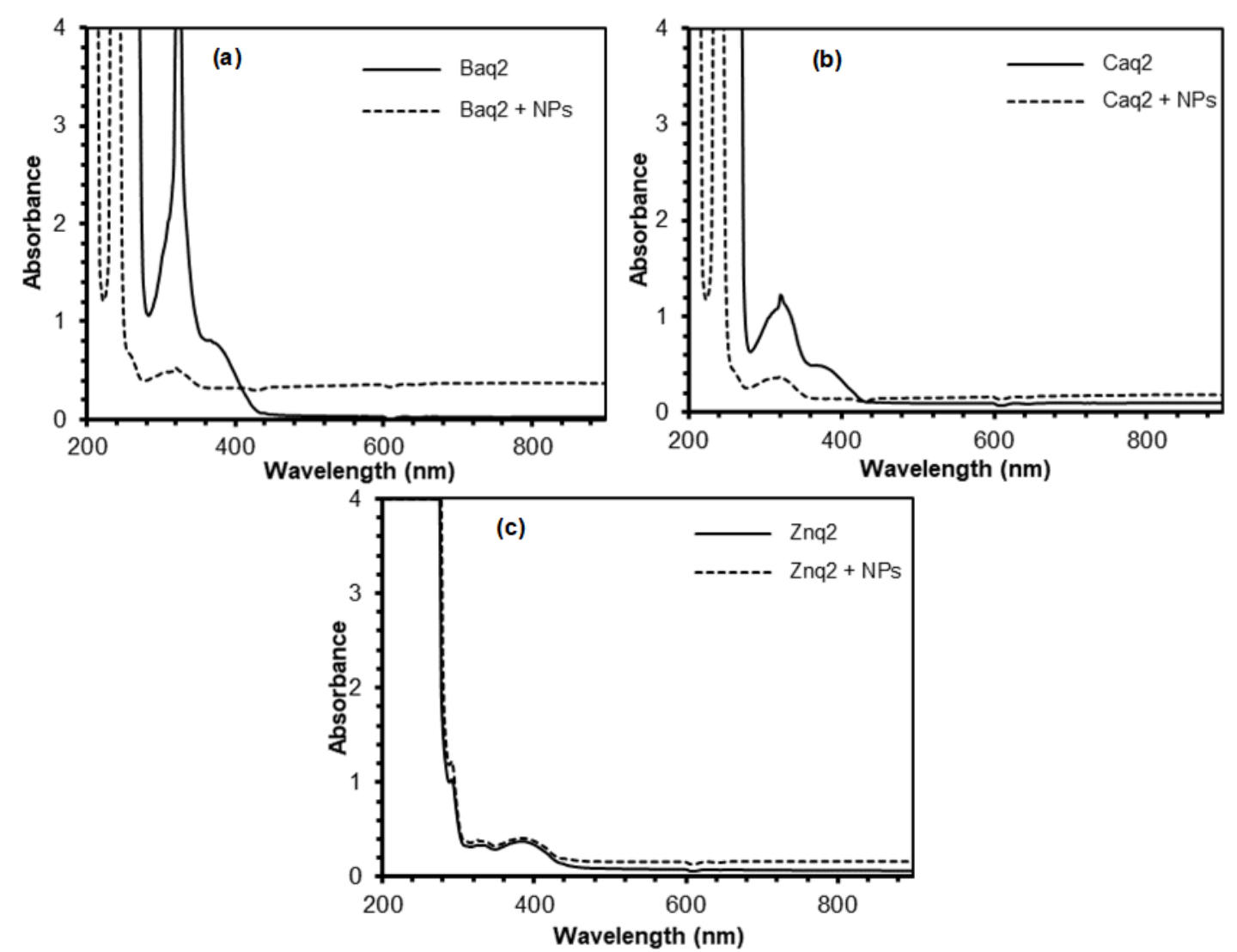

Fig 2. Absorption spectra of $\mathrm{Baq}_{2}$ complex (a), $\mathrm{Caq}_{2}$ complex (b), and $\mathrm{Znq}_{2}$ complex (c) prepared in this work before and after adding nanoparticles

nanoparticles to the complex solution in ethanol solvent. It is clear that the absorption peak for $\mathrm{Baq}_{2}$ and $\mathrm{Caq}_{2}$ complexes at 326 and $330 \mathrm{~nm}$, respectively, was suppressed after adding the nanoparticles, while no similar effect was observed for the $\mathrm{Znq}_{2}$ complex. However, after adding the nanoparticles, all complexes showed high absorbance in the ultraviolet region $(<300 \mathrm{~nm})$. As well, the $\mathrm{Baq}_{2}$ complex showed higher absorbance in the spectral range 500-800 $\mathrm{nm}$ after adding the nanoparticles, while the difference in absorbance for both $\mathrm{Caq}_{2}$ and $\mathrm{Znq}_{2}$ complexes was very small.

As the aimed application of the organometallic complexes prepared in this work is the random gain media, then the photoluminescence intensities were compared before and after adding the nanoparticles, as shown in Fig. 3. All complexes showed two distinct peaks; the first in the blue region (450-470 nm) and the second in the green region (510-515 nm). In the $\mathrm{Baq}_{2}$ and $\mathrm{Caq}_{2}$ complexes, the intensity of the first peak was higher than that of the second one. In contrast, the second peak of the $\mathrm{Znq}_{2}$ was higher than that of the first one.

After adding the nanoparticles, the $\mathrm{Caq}_{2}$ complex showed a slight decrease in the photoluminescence intensity, whereas both $\mathrm{Baq}_{2}$ and $\mathrm{Znq}_{2}$ complexes showed higher photoluminescence intensities. The increase for the $\mathrm{Znq}_{2}$ complex was $25 \%$ and $20 \%$ for both peaks, while it was more than $2 \%$ and $9 \%$ for the $\mathrm{Baq}_{2}$ complex.

The FE-SEMs (Fig. 4) for both samples before nanoparticles addition were measured to attribute the different increases in photoluminescence for $\mathrm{Baq}_{2}$ and $\mathrm{Znq}_{2}$ complexes. The SEM was not performed on the synthesized samples after adding $\mathrm{TiO}_{2}$ nanoparticles as no reaction is expected between the complex and nanoparticles. Instead, the SEM result of $\mathrm{TiO}_{2}$ nanoparticles was inserted to confirm their formation. As a result, it was confirmed that the metal ions linked to the ligand form nanoparticles. As shown, the $\mathrm{Baq}_{2}$ sample 


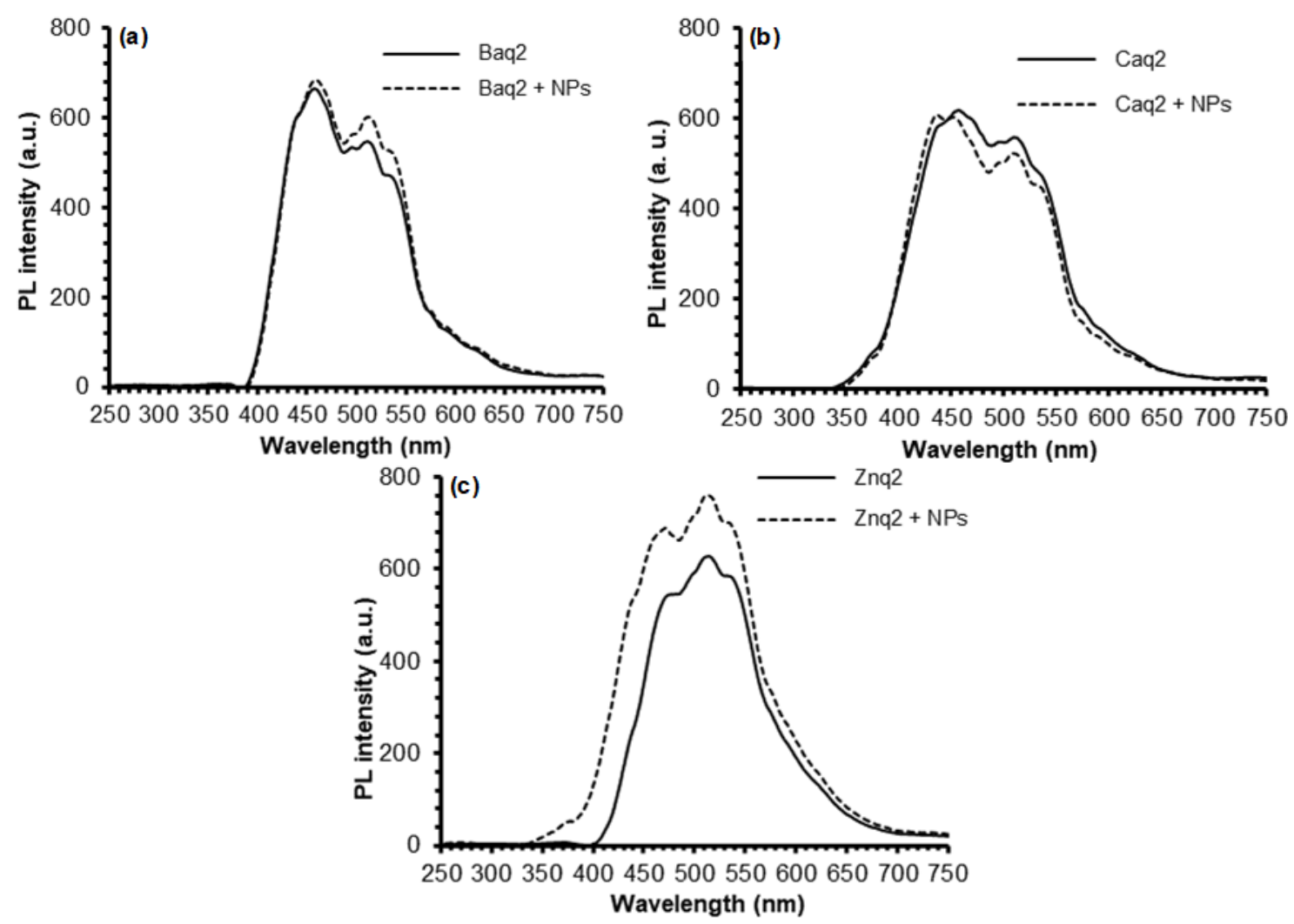

Fig 3. Photoluminescence spectra of $\mathrm{Baq}_{2}$ complex (a), $\mathrm{Caq}_{2}$ complex (b), and $\mathrm{Znq}_{2}$ complex (c) prepared in this work before and after adding nanoparticles
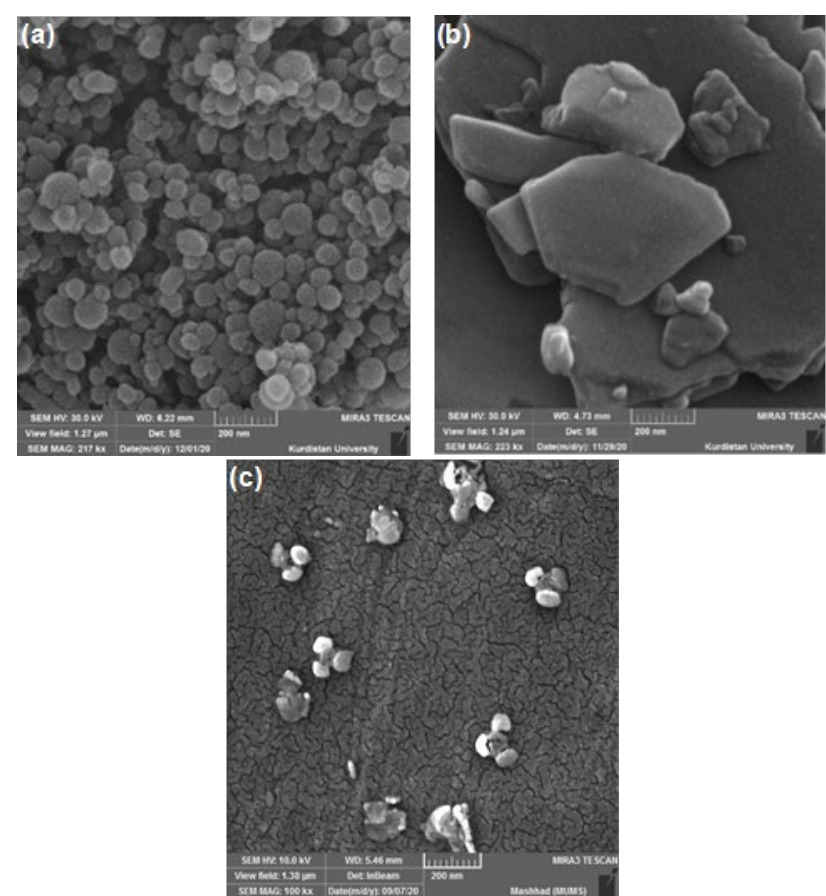

Fig 4. FE-SEM results of $\mathrm{Baq}_{2}$ complex (a) and $\mathrm{Znq}_{2}$ complex (b) prepared in this work before adding nanoparticles (c) SEM result of $\mathrm{TiO}_{2}$ nanoparticles exhibited a highly uniform distribution, sphericalshaped, and smaller particles when compared to the $\mathrm{Znq}_{2}$ sample, which exhibited larger sizes and reasonable aggregation. The homogeneous distribution of nanoparticles may cause a homogeneous response to the incident radiation, and hence the average increase is smaller than that in the case of the inhomogeneous distribution of the $\mathrm{Znq}_{2}$ sample.

Accordingly, the $\mathrm{Znq}_{2}$ complex can be a good candidate for a random gain medium to produce a random laser. Furthermore, with further optimization of preparation conditions, mainly the concentrations of complex solution and nanoparticles added to the complex solution, the photoluminescence characteristics can be sufficiently enhanced to fabricate the final sample as a solid rod by embedding the sample in a transparent host.

\section{- CONCLUSION}

In concluding remarks, organometallic complexes containing nanoparticles were prepared from the 8- 
hydroxyquinoline ligand linked to metal ions such as $\mathrm{Ba}^{2+}$, $\mathrm{Ca}^{2+}$, and $\mathrm{Zn}^{2+}$. The $\mathrm{Znq}_{2}$ complex showed constant absorbance after adding the nanoparticles but higher photoluminescence intensity in the visible region. The random gain media made from the $\mathrm{Znq}_{2}$ complex with nanoparticles showed the best characteristics with good chemical and spectroscopic stabilities, high reliability, and reproducibility in addition to the low production cost and reasonably simple requirements.

\section{- AUTHOR CONTRIBUTIONS}

All authors agreed to the final version of this manuscript.

\section{- REFERENCES}

[1] Nasser, B.K., and Hameed, M.A., 2020, Narrow emission linewidth of highly-pure silicon nitride nanoparticles in different dye solutions as random gain media, Nonlinear Opt., Quantum Opt., 53 (1-2), 99-105.

[2] Chiad, B.T., Latif, K.H., Kadhim, F.J., and Hameed, M.A., 2011, Random laser of $\mathrm{R} 6 \mathrm{G}$ dye and $\mathrm{TiO}_{2}$ nanoparticles doped in PMMA polymer, Adv. Mater. Phys. Chem., 1, 20-25.

[3] Chiad, B.T., Hameed, M.A., Latif, K.H., and Al-Maliki, F.J., 2011, Transition from amplified spontaneous emission to laser action in disordered media of R6G dye and $\mathrm{TiO}_{2}$ nanoparticles doped with PMMA polymer, J. Eur. Opt. Soc.-Rapid Publ., 6, 11049.

[4] Liu, J., Zhong, X., Xu, Y., and Li, Y., 2019, Green synthesis of 8-hydroxyquinoline barium as visiblelight-excited luminescent material using mechanochemical activation method, Global Challenges, 3 (12), 1900052.

[5] Dai, G., Wang, L., and Deng, L., 2020, Flexible random laser from dye doped stretchable polymer film containing nematic liquid crystal, Opt. Mater. Express, 10 (1), 68-75.

[6] Liu, J., Zhang, H., Dong, H., Meng, L., Jiang, L., Wang, Y., Yu, J., Sun, Y., Hu,W., and Heeger, A.J., 2015, High mobility emissive organic semiconductor, Nat. Commun., 6 (1), 10032.
[7] Wang, W., Marshall, M., Collins, E., Marquez, S., $\mathrm{Mu}$, C., Bowen, K.H., and Zhang X., 2019, Intramolecular electron-induced proton transfer and its correlation with excited-state intramolecular proton transfer, Nat. Commun., 10 (1), 1170.

[8] Wu, S., Zhong, X., Zeng, H., You, W., and Zhou, W., 2018, Study on green synthesis and properties of luminescent material bis(8-hydroxyquinoline) calcium $\left(\mathrm{CaQ}_{2}\right)$, J. Lumin., 195, 120-125.

[9] Prachayasittikul, V., Prachayasittikul, S., Ruchirawat, S., and Prachayasittikul V., 2013, 8Hydroxyquinolines: A review of their metal chelating properties and medicinal applications, Drug Des., Dev. Ther., 7, 1157-1178.

[10] Albrecht, M, Fiege, M., and Osetska, O., 2008, 8Hydroxyquinolines in metallosupramolecular chemistry, Coord. Chem. Rev., 252 (8-9), 812-824.

[11] El-Wakiel, N.A., Rizk, H.F., and Ibrahim, S.A., 2017, Synthesis and characterization of metal complexes of azo dye based on 5-nitro-8-hydroxyquinoline and their applications in dyeing polyester fabrics, Appl. Organometal. Chem., 31 (10), e3723.

[12] Vashi, R.T., Patel, S.B., and Kadiya, H.K., 2012, Synthesis, characterization and antimicrobial activity of metal chelates of 2-[(8-hydroxyquinolinyl)-5aminomethyl]-3-(4-bromophenyl)-3(H)-quinazolin -4-one, Pharma Chem., 4 (4), 1506-1511.

[13] Mahmood, A.A., Hammadi, O.A., and Ibraheem, K.R., 2021, Some physical properties of metalhydroxyquinoline complexes in different solvents, Iraqi J. Appl. Phys., 17 (1), 9-16.

[14] Lima, C.F.R.A.C., Taveira, R.J.S., Costa, J.C.S., Fernandes, A.M., Melo, A., Silva, A.M.S., and Santos, L.M.N.B.F., 2016, Understanding M-ligand bonding and mer-/fac-isomerism in tris(8hydroxyquinolinate) metallic complexes, Phys. Chem. Chem. Phys., 18 (24), 16555-16565.

[15] Podunavac-Kuzmanovic, S.O., Cvetkovic, D.M., and Vojinovic, L.S., 2004, Synthesis, physicochemical characterization and biological activity of 2-aminobenzimidazole complexes with different metal ions, Acta Period. Technol., 35, 239-246. 
[16] Han, Y.K., and Lee, S.U., 2002, Molecular orbital study on the ground and excited states of methyl substituted tris(8-hydroxyquinoline) aluminum(III), Chem. Phys. Lett., 366 (1-2), 9-16.

[17] Wagenknecht, P.S., and Ford, P.C., 2011, Metal centered ligand field excited states: Their roles in the design and performance of transition metal based photochemical molecular devices, Coord. Chem. Rev., 255 (5-6), 591-616.

[18] Pimchan, P., Khaorapapong, N., and Ogawa, M., 2014, The effect of acetyl trimethyl ammonium ion and type of smectites on the luminescence efficiency of bis(8-hydroxyquinoline)zinc(II) complex, Appl. Clay Sci., 101, 223-228.

[19] Tsuboi, T., Nakai, Y., and Torii, Y., 2012, Photoluminescence of bis(8-hydroxyquinoline) zinc $\left(\mathrm{Znq}_{2}\right)$ and magnesium $\left(\mathrm{Mgq}_{2}\right)$, Cent. Eur. J. Phys., 10 (2), 524-528.

[20] Patel, K.D., and Patel, H.S., 2017, Synthesis, spectroscopic characterization and thermal studies of some divalent transition metal complexes of 8hydroxyquinoline, Arabian J. Chem., 10, S1328S1335. 